\title{
Impacto de la pandemia COVID-19 en la práctica de la electrofisiología cardíaca en Latinoamérica: Una encuesta de la Sociedad Latinoamericana del Ritmo Cardíaco
}

\author{
Alejandro Cuesta', Manlio F. Márquez-Murillo², Luis C. Saenz³', \\ Marcio Jansen De Oliveira-Figueiredo ${ }^{4}$
}

\section{Resumen}

$\mathrm{Al}$ comienzo de la pandemia COVID-19 se implementaron pautas clínicas restrictivas de la asistencia que incluyeron los Servicios de Electrofisiología (SEF).

Objetivo: analizar la actividad asistencial y conocer la situación de los SEF en Latinoamérica a dos meses de iniciadas las restricciones.

Método: estudio observacional descriptivo-analítico y transversal, utilizando una encuesta a médicos electrofisiólogos en marzo/2020. Se comparó la actividad clínica e invasiva que se realizaba antes y durante la pandemia.

Resultados: se incluyeron 147 encuestas, de 74 ciudades y 18 países de Latinoamérica. Los actos clínicos semanales se redujeron de $75(45 / 127)$ a $20(10 / 40)(\mathrm{p}<0,001)$, bajaron $71 \%$. Los procedimientos invasivos mensuales se redujeron de $26(13 / 39)$ a $4(2 / 9)(p<0,001)$, bajaron $77 \%$. El $49 \%$ encuestado trabajaban en $\geq 3$ centros asistenciales y $89 \%$ compartía laboratorio con un servicio de hemodinamia. La ocupación de camas en los hospitales era baja $37 \%$, intermedia $28 \%$ y alta $35 \%$. El 30\% refirió algún médico de su equipo fue puesto en cuarentena por infección/ contacto. El 53\% refirió no se hacía ninguna prueba de tamizaje a los pacientes previa a los procedimientos y $77 \%$ al personal. La mayoría percibía dificultades como importantes o muy importantes, pero $63 \%$ estaba considerando la reapertura al funcionamiento normal.

Conclusiones: hubo una reducción importante de la actividad clínica e invasiva. La mayoría no tenía alta ocupación de camas. Los encuestados trabajaban en varios centros y en salas de hemodinamia. Aún no se habían implementado totalmente las medidas de prevención. Existía la percepción de que en poco tiempo se retomaría la normalidad.

Palabras clave: PANDEMIA

COVID-19

ELECTROFISIOLOGÍA

PAUTAS CLÍNICAS

1. Centro Cardiovascular Universitario, Hospital de Clínicas, Universidad de la República, Montevideo, Uruguay

2. Unidad Coronaria, Instituto Nacional de Cardiología “Ignacio Chávez”, Ciuidad de México, México

3. Centro Internacional de Arritmias, Fundación Cardio Infantil, Bogotá, Colombia

4. Cardiología, Servicio de Electrofisiología, Universidad de Campinas, (UNICAMP), Campinas, Brazil.

Correspondencia: Dr. Alejandro Cuesta MD PhD. Correo electrónico: arritmia@yahoo.com

Los autores declaran no tener ningún conflicto de intereses en relación al tema de este artículo, más que el que deviene de su condición profesional y de especialistas en el área.

La presente investigación no ha recibido ninguna beca específica de agencias de los sectores públicos, comerciales o privados sin fines de lucro. Fue realizado con recursos propios de la Sociedad Latinoamericana del Ritmo Cardíaco (LAHRS).

Recibido Set 6, 2021; aceptado Oct 29, 2021 
Impact of the COVID-19 pandemic on the practice of cardiac electrophysiology in Latin America: A Survey of the Latin American Heart Rhythm Society

\section{Summary}

At the beginning of the COVID-19 pandemic, restrictive clinical guidelines were implemented, including Electrophysiology Services (EFS).

Objectives: analyze the healthcare activity and to know the situation of the EFS in Latin America two months after the restrictions began.

Method: descriptive-analytical and cross-sectional observational study, using a survey of electrophysiologists in March / 2020. The clinical and invasive activity carried out before and during the pandemic was compared.

Results: 147 surveys were included, from 74 cities in 18 Latin American countries. Weekly clinical events were reduced from $75(45 / 127)$ to $20(10 / 40)(\mathrm{p}<0.001)$, they fell $71 \%$. Monthly invasive procedures were reduced from $26(13 / 39)$ to $4(2 / 9)(\mathrm{p}<0.001)$, down $77 \%$. Forty-nine percent surveyed worked in $\geq 3$ healthcare centers and $89 \%$ shared a laboratory with a hemodynamic service. Hospital bed occupancy was low $37 \%$, intermediate $28 \%$ and high $35 \%$. Thirty percent referred a doctor from their team was quarantined for infection / contact. Fifty-three percent reported that no screening test was done on the patients prior to the procedures and $77 \%$ on the staff. Most perceived difficulties as important or very important, but $63 \%$ were considering reopening to normal functioning.

Conclusion: There was a significant reduction in clinical and invasive activity. Most did not have high bed occupancy. Respondents worked in various centers and in hemodynamic rooms. Prevention measures had not yet been fully implemented yet. There was a perception that in a short time normality would return.

Key words: $\quad$ Pandemic

COVID-19

Electrophysiology

Clinical guidelines

Impacto da pandemia COVID-19 na prática da eletrofisiologia cardíaca na América Latina: uma pesquisa da Sociedade Latino-Americana de Ritmo Cardíaco

\section{Resumo}

No início da pandemia de COVID-19 foram implementadas diretrizes clínicas, incluindo os Serviços de Eletrofisiologia (SEF).

Objetivo: analisar a atividade assistencial e conhecer a situação do SEF na América Latina dois meses após o início das restrições.

Método: estudo descritivo-analítico e observacional transversal, por meio de questionário com eletrofisiologistas em março / 2020. Foi comparada a atividade clínica e invasiva realizada antes e durante a pandemia.

Resultados: foram incluídos 147 inquéritos, de 74 cidades e 18 países latino-americanos. O número de procedimentos semanais foi reduzido de $75(45 / 127)$ para $20(10 / 40)(\mathrm{p}<0,001)$, com redução de $71 \%$. Os procedimentos invasivos mensais foram reduzidos de 26 (13/39) para 4 (2/9) ( $p<0,001$ ), com redução de 77\%. Dos eletrofisiologistas que responderam ao questionário, $49 \%$ trabalhavam em 3 ou mais centros, e $89 \%$ compartilhavam o laboratório com serviço de hemodinâmica. A ocupação de leitos hospitalares foi baixa em $37 \%$, intermediária em $28 \%$ e alta em $35 \%$. Dos que responderam al questionário, 30\% relataram que um médico de sua equipe foi colocado em quarentena por infecção ou contato. Foi relatado que, dentre os que responderam, $53 \%$ não realizava teste de triagem nos pacientes antes dos procedimentos, e em $77 \%$ na equipe. A maioria percebeu as dificuldades como importantes ou muito importantes, mas $63 \%$ consideravam a reabertura ao funcionamento.

Conclusões: houve redução significativa da atividade clínica e invasiva. A maioria não tinha grande ocupação de leitos. Os entrevistados trabalhavam em vários centros e em salas de hemodinâmica. As medidas de prevenção ainda não haviam sido totalmente implementadas, porém havia a percepção de que em pouco tempo a normalidade voltaria.

Palavras chave: Pandemia

COVID-19

Eletrofisiologia

Diretrizes Clínicas 
¿Qué aporta este estudio al conocimiento actual?

Este estudio aporta información cuantitativa sobre cómo se vio comprometida la atención de pacientes en los servicios de electrofisiología al inicio de la pandemia COVID-19. Agrega el contexto de ocupación de los centros asistenciales, afectación del personal y las medidas que se tenían implementadas para evitar contagios. Informa también sobre las dificultades que se percibían para retomar la actividad normal y a pesar de ello, la expectativa de una pronta resolución.

\section{Introducción}

La pandemia COVID 19 determina una gran carga a la morbilidad y mortalidad. La infección por SARS-CoV-2 tiene como características más alarmantes, en relación a otros virus, su alta tasa de contagio y mortalidad. La aparición de un gran número de casos en un corto tiempo ha llevado a superar la capacidad de atención de muchos países $^{(1)}$. La infección por SARS-CoV-2 se puede presentar con síntomas similares a ciertas enfermedades cardiovasculares. Las personas con factores de riesgo cardiovasculares tienen más chances de sufrir la enfermedad viral y tienen una evolución más desfavorable ${ }^{(2,3)}$. Por otro lado, los pacientes ingresados por una enfermedad cardiovascular, pueden adquirir la enfermedad al ser hospitalizados en centros asistenciales que atiende pacientes con COVID-19 y por ello agravar su evolución. Por esas razones, se implementaron medidas restrictivas para la asistencia de todos los pacientes y ello incluyó a los Servicios de Electrofisiología (SEF). Sociedades norteamericanas en conjunto y la Sociedad Latinoamericana del Ritmo Cardíaco (LAHRS) fueron las primeras en publicar pautas al respecto ${ }^{(4-6)}$. Los objetivos generales de los protocolos de asistencia a nivel hospitalario buscaron evitar el contagio, tanto de pacientes como del personal de salud, y reservar capacidad locativa, recursos humanos y materiales para asistir pacientes con COVID-19.

Entre las medidas adoptadas se incluyeron la suspensión de consultas ambulatorias presenciales, de realización de exámenes complementarios y de estudios de coordinación. También se estableció la suspensión de procedimientos electrofisiológicos invasivos e implantes de dispositivos que pudieran diferirse. La adherencia a los protocolos en los SEF no ha sido analizada, ni su consecuencia sobre la asistencia.

Cuando se hizo la fase de campo de este estudio, en Sudamérica habían 127 fallecidos y 2000 casos por millón de habitantes. La evolución ha sido en descensos y ascensos de incidencia, que han obligado a los sistemas de salud a volver a tomar medidas que semanas antes suspendían. Ahora nos enfrentamos a nuevas variantes del SARS-CoV-2 con mayor poder de contagio. Parece muy importante evaluar lo realizado, aprender de los errores, estar mejor preparados y tomar mejores decisiones en el futuro.

Los objetivos de este estudio fueron: 1) Evaluar la disminución de la actividad asistencial de los SEF, clínica e invasiva, a los dos meses de iniciadas las restricciones impuestas por COVID-19 en Latinoamérica. 2) Conocer la situación de los SEF en relación al centro hospitalario donde asienta, la disponibilidad de equipos de protección personal y la asistencia en la especialidad de pacientes COVID-19. 3) Investigar cuáles son las dificultades más importantes previstas por los médicos especialistas para retomar luego las actividades normales.

\section{Material y métodos}

Se realizó un estudio observacional descriptivo-analítico y transversal.

Población: se incluyeron médicos que se desempeñan en SEF cardíaca en países de Latinoamérica independientemente de la complejidad del servicio o del tamaño de la población de referencia.

Diseño: se realizó una encuesta auto administrada de 28 preguntas cerradas, a través de un formulario en línea, confeccionado en la plataforma comercial SurveyMonkey®. Se registró la ciudad y el país de origen, el número de actos médicos en unidad de tiempo que se estaba realizando y que se realizaba previo a la pandemia, características de los mismos, características del hospital, contacto con pacientes COVID-19 y la disponibilidad de materiales. No se condicionó la forma en que cada encuestado recababa los números de su servicio. Se ofreció una lista cerrada de dificultades para el inicio de la actividad normal, cuya importancia relativa se debía calificar en una escala cualitativa. Los encuestados podían dejar preguntas en blanco salvo la procedencia. El formulario fue evaluado previamente entre médicos cardiólogos. Se incluyó una pregunta para verificar que el encuestado era médico electrofisiólogo. Si no lo era y/o no se contestaba 50\% del formulario no fue incluido en el análisis.

La convocatoria a llenar la encuesta se realizó a través de la lista de socios activos de la Sociedad Latinoamericana del Ritmo Cardíaco (LAHRS) y 


\begin{tabular}{|lcc|}
\hline \multicolumn{3}{l}{ Tabla 1. Número de encuestados por países. } \\
\hline País & Encuestados & $\%$ \\
\hline Brasil & 33 & 22,4 \\
Colombia & 29 & 19,7 \\
\hline México & 25 & 17,0 \\
\hline Argentina & 21 & 14,3 \\
\hline Venezuela & 6 & 4,1 \\
\hline Uruguay & 5 & 3,4 \\
\hline República Dominicana & 5 & 3,4 \\
\hline Chile & 4 & 2,7 \\
\hline Ecuador & 4 & 2,7 \\
\hline Peru & 3 & 2,0 \\
\hline Bolivia & 2 & 1,4 \\
\hline El Salvador & 2 & 1,4 \\
\hline Honduras & 2 & 1,4 \\
\hline Panamá & 2 & 1,4 \\
\hline Guatemala & 1 & 0,7 \\
\hline Nicaragua & 1 & 0,7 \\
\hline Panamá & 1 & 0,7 \\
\hline Puerto Rico & 147 & 0,7 \\
\hline Total = 18 & & 100,0 \\
\hline
\end{tabular}

estuvo disponible durante las 3 últimas semanas del mes de mayo de 2020. En esas fechas ya habían transcurrido dos meses del inicio de las medidas de prevención en la mayoría de los países, si bien ello fue heterogéneo. Su llenado no era obligatorio ni se estimulaba la participación con ningún bien económico o material. Tenía una versión en idioma portugués y otra en castellano.

\section{Análisis estadístico}

Las variables continuas con distribución normal se expresan en media \pm 1 DS y compararon previo y durante la pandemia con test de T para muestras apareadas. Las variables continuas no paramétricas se expresan en mediana y cuartiles $25 / 75$ y se compararon con test de Wilcoxon. Las variables nominales se describen mediante frecuencia absoluta, relativa y se compararon con test de McNemar. Se consideró significativa una $\mathrm{p}<0,05$.

\section{Resultados}

Se recibieron 159 encuestas no repetidas. Se eliminaron 12 por los criterios de exclusión establecidos, 8 por no completar $50 \%$ y 4 por error en la respuesta control. Se analizaron 147 encustadas; $23(16 \%)$ completadas en portugués y 124 (84\%) en castellano (figura 1). Los electrofisiólogos en-

\section{Universo:}

350 Electrofisiólogos registrados

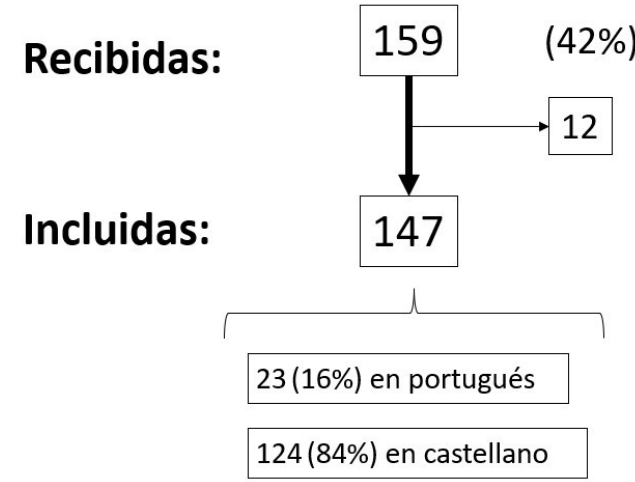

Figura 1. Universo y número de encuestas recibidas e incluidas.

cuestados se desempeñaban en 74 ciudades de 18 países de Latinoamérica y su distribución se muestra en la tabla 1. La edad de los encuestados fue $48 \pm 17$ años.

\section{Actividad de los servicios}

En la tabla 2 se muestra el número de actos clínicos semanales presenciales y remotos que se realizaban previo y durante la pandemia. El conjunto de todos los tipos de actos incluidos tuvo una reducción de $75(45 / 127)$ a $20(10 / 40)(\mathrm{p}<0.001)$. Esto constituyó un descenso global de la actividad clínica semanal de 71\%. Habiendo un aumento de las consultas clínicas a distancia, pero no del control de dispositivos a distancia. (figura 2).

En la tabla 3 se muestra el número de procedimientos invasivos mensuales que se realizaban previo y durante a la pandemia. Hubo una reducción significativa del número absoluto de todos los tipos de procedimientos incluidos. El número total de procedimientos mensuales bajó de 26 (13/39) a $4(2 / 9)$. Esto constituyó una reducción global de $77 \%$ (figura 2). Antes de la pandemia el implante de Marcapasos era el 39\% de los procedimientos mensuales y durante la pandemia subió al 54\%.

La oportunidad de los procedimientos antes y durante se muestran en la tabla 4 expresados en media y desvío del porcentaje referido por cada encuestado. Previo a la pandemia el 51\% de los procedimientos eran realizados de coordinación y $23 \%$ de emergencia, durante la pandemia pasó a ser $6 \%$ y $66 \%$ respectivamente.

\section{Situación de los servicios}

El 43\% (63) de los encuestados trabajaba solo en 


\begin{tabular}{|c|c|c|c|c|c|}
\hline \multirow[t]{2}{*}{ Cantidad /semana } & \multicolumn{2}{|c|}{ Previo COVID-19 } & \multicolumn{2}{|c|}{ Durante COVID-19 } & \multirow[t]{2}{*}{$p$} \\
\hline & $M$ & $25 / 75$ & $M$ & $25 / 75$ & \\
\hline Consultas clínicas & 30 & $20 / 50$ & 3 & $0 / 6$ & $<0,001$ \\
\hline Consultas clínicas a distancia & 0 & $0 / 2$ & 5 & $0 / 10$ & $<0,001$ \\
\hline Control de dispositivos & 15 & $8 / 32$ & 2 & $1 / 5$ & $<0,001$ \\
\hline $\begin{array}{l}\text { Control de dispositivos } \\
\text { a distancia }\end{array}$ & 0 & $0 / 2$ & 0 & $0 / 1$ & NS \\
\hline Estudios Holter & 12 & $5 / 33$ & 2 & $0 / 7$ & $<0,001$ \\
\hline Mesa basculante & 0 & $1 / 4$ & 0 & $0 / 0$ & $<0,001$ \\
\hline Toda actividad clínica & 75 & $45 / 127$ & 20 & $10 / 40$ & $<0,001$ \\
\hline
\end{tabular}

Tabla 3. Cantidad de procedimentos invasivos mensuales que se realizaban previo y durante la pandemia COVID-19, por rubro y comparada.

\begin{tabular}{|c|c|c|c|c|c|}
\hline \multirow[t]{2}{*}{ Cantidad / mes } & \multicolumn{2}{|c|}{ Previo COVID-19 } & \multicolumn{2}{|c|}{ Durante COVID-19 } & \multirow[t]{2}{*}{$p$} \\
\hline & $M$ & $25 / 75$ & $M$ & $25 / 75$ & \\
\hline Implantes de MPD & 10 & $5 / 16$ & 2 & $1 / 5$ & $<0,001$ \\
\hline Implantes de DAI & 2 & $1 / 4$ & 0 & $0 / 1$ & $<0,001$ \\
\hline Implantes de TRC & 1 & $1 / 2$ & 0 & $0 / 1$ & $<0,001$ \\
\hline Extracción de electrodos & 0 & $0 / 1$ & 0 & $0 / 0$ & $<0,001$ \\
\hline Ablaciones TPSV convencionales & 5 & $3 / 10$ & 0 & $0 / 2$ & $<0,001$ \\
\hline Ablaciones fibrilación auricular & 2 & $0 / 4$ & 0 & $0 / 0$ & $<0,001$ \\
\hline Ablaciones EV/TV & 1 & $0 / 2$ & 0 & $0 / 0$ & $<0,001$ \\
\hline Total de procedimientos / mes & 26 & $13 / 39$ & 4 & $2 / 9$ & $<0,001$ \\
\hline
\end{tabular}

MPD: marcapasos definitivo; DAI: desfibrilador automático implantable; TRC: terapia de resincronización cardíaca; TPSV: taquicardia paroxística supraventricular; EV/TV extrasístoles y taquicardias ventriculares; M: Mediana; 25/75: cuartiles $25 \%$ y $75 \%$ de la distribución

centros privados, $10 \%$ (15) solo en centros públicos y $47 \%$ (69) en ambos tipos. El $49 \%$ trabajaba habitualmente en 3 o más centros asistenciales.

La ocupación de camas en los hospitales en que se desempeñaban fue referida como baja en $37 \%$ (55), intermedia en $28 \%$ (41) y alta o muy alta en $35 \%$ (51).

El 92\% de los encuestados trabajaba en un hospital que estaba abierto a la recepción de pacientes con COVID-19 sospechado o confirmado.

El 89\% (131) de los electrofisiólogos encuestados compartían la sala de procedimientos con el servicio de hemodinamia y $23 \%$ (34) dijeron que se habían realizado procedimientos a pacientes con COVID-19 en la sala donde trabajaban.

El 33\% (49) de los encuestados había realizado al menos una consulta clínica a pacientes con COVID-19 confirmado o con alta sospecha y $8 \%$ (12) había realizado al menos un procedimiento invasivo.

El 30\% (44) refirió que él o algún médico de su equipo habían sido puestos en cuarentena por

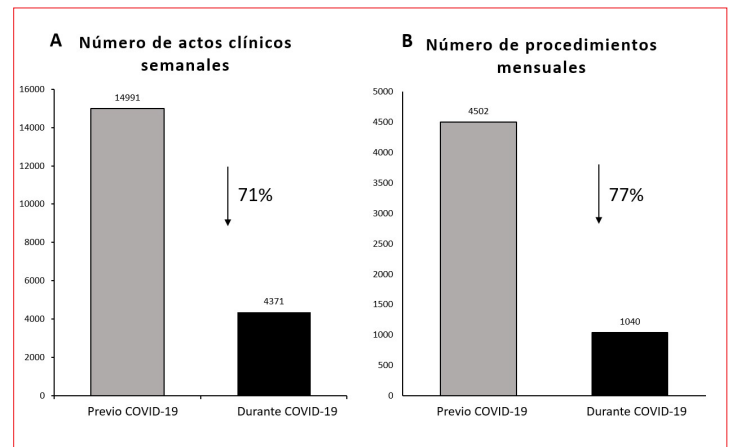

Figura 2. Histogramas de frecuencias absolutas de (A) actos clínicos semanales y de (B) procedimientos mensuales en servicio de electrofisiología de Latinoamérica, previo y durante la pandemia COVID19.

infección con SARS-CoV-2 o por contacto con persona confirmada.

\section{Protocolos de protección y prevención}

Al momento que se realizó la encuesta el 67\% (98) de los médicos refirieron que la adherencia a los 


\begin{tabular}{|c|c|c|c|}
\hline & $\begin{array}{c}\text { Previo COVID-19 } \\
(M \pm D S)\end{array}$ & $\begin{array}{c}\text { Durante COVID-19 } \\
(M \pm D S)\end{array}$ & $p$ \\
\hline Procedimientos de emergencia & $23 \pm 23$ & $66 \pm 37$ & $<0,001$ \\
\hline Procedimientos de urgencia & $26 \pm 20$ & $19 \pm 27$ & 0,027 \\
\hline Procedimientos de coordinación & $51 \pm 27$ & $6 \pm 15$ & $<0,001$ \\
\hline
\end{tabular}

Tabla 5. Preguntas referentes a las medidas de protección personal y prevención de contagios intra hospitalarios del personal y pacientes.

$$
\text { Pregunta }
$$

$n$

$(\%)$

¿Qué adherencia pudo tener a los protocolos internacionales de protección personal para realizar procedimientos?

$\begin{array}{lcc}\text { Alta } & 98 & (67) \\ \text { Intermedia } & 41 & (28) \\ \text { Baja } & 8 & (5)\end{array}$

¿Se realiza alguna prueba a todos los pacientes previo a los rocedimientos?

$\begin{array}{lcc}\text { Estudio serológico } & 9 & \text { (6) } \\ \text { Hisopado / PCR } & 60 & (41) \\ \text { Ninguna } & 78 & \text { (53) }\end{array}$

¿Se realiza algún estudio de tamizaje al personal de salud en su hospital principal?

$$
\text { NO }
$$

113

¿Hay definida una ruta de circulación de pacientes COVID-19 en su hospital principal?

$$
\mathrm{SI}
$$$$
128
$$

protocolos internacionales de protección personal para realizar procedimientos era alta, el resto consideró que era media o baja (tabla 5).

El 53\% (78) de los encuestados refirió que previo a los procedimientos no se hacía ninguna prueba de tamizaje a los pacientes que no fueran sospechosos y 77\% (113) que no se hacía ninguna prueba de tamizaje al personal en el hospital principal en que trabajaba.

El 87\% (128) dijo se habían definido rutas de circulación de pacientes COVID-19 en su hospital principal.

\section{Regreso al funcionamiento normal}

Al momento de ser encuestados 63\% (92) estaban considerando la reapertura al funcionamiento normal del servicio. Las dificultades percibidas para ello fueron ponderadas de la manera que se muestra en la tabla 6. Fue evaluado como muy importante, el temor de los pacientes a realizarse procedimientos que requirieran hospitalización por un 51\% y la demora en recuperar el volumen de consultas y exámenes ambulatorios un 38\%. Estas fueron las que recibieron las ponderaciones más altas. La que fue evaluada con la menor jerarquía por más participantes fue la falta de rutas hospitalarias para evitar contagios cruzados COVID-19 (27\%).

\section{Discusión}

En el momento en que se hizo la encuesta los participantes refirieron una reducción muy importante de los actos clínicos semanales presenciales realizados por los SEF (tabla 2). Hubo un aumento de las consultas clínicas remotas, pero fue muy inferior y no llegó a compensar el descenso. Esto pudo haber ocurrido por los protocolos sanitarios establecidos y también por la sola voluntad de pacientes y del personal.

No hubo un aumento significativo del control remoto de dispositivos implantados. Previo al inicio de la pandemia ya era utilizado por muy pocos encuestados y en números pequeños. En los meses posteriores se publicaron pautas mundiales al respecto, con la participación de $\mathrm{LAHRS}^{(6)}$. No sabemos cómo evolucionó la incorporación de esa práctica ni la adquisición de la tecnología necesaria para su instrumentación. Esa posibilidad es muy dependiente de lo económico, que debe ser subsanada entre los servicios de salud, los financiadores y la industria.

Hubo un descenso muy importante de todos los tipos de procedimientos invasivos (77\%) (tabla 3). El descenso en las ablaciones fue similar a lo ocurrido en algunas ciudades de Europa y China en que también fue analizado ${ }^{(7)}$. Las pocas ablaciones que se siguieron haciendo allí fueron principalmente de urgencia o emergencia, lo mismo que encontramos en esta encuesta (tabla 4) ${ }^{(7)}$. Esto explica porque el implante de marcapasos pasó a tener una importancia relativa mayor en el total de procedimientos durante la pandemia. Son las intervenciones que más frecuentemente se deben realizar de urgencia.

El descenso tan importante de la actividad invasiva en los SEF en Europa y China ocurrió cuando los países estaban en franco ascenso y 


\begin{tabular}{|c|c|c|c|c|c|}
\hline \multirow[t]{2}{*}{ Dificultad percibida para volver al funcionamiento normal } & \multicolumn{2}{|c|}{ Nada importante } & \multirow{2}{*}{$\begin{array}{c}n(\%) \\
3\end{array}$} & \multicolumn{2}{|c|}{ Muy importante } \\
\hline & 1 & 2 & & 4 & 5 \\
\hline $\begin{array}{l}\text { Demora en la recuperación de los volúmenes de consulta } \\
\text { ambulatoria y realización de exámenes diagnósticos. }\end{array}$ & $14(10)$ & $11(7)$ & $24(16)$ & $42(29)$ & $56(38)$ \\
\hline $\begin{array}{l}\text { Temor de los pacientes de realizarse procedimientos que re- } \\
\text { quieran hospitalización por miedo a contraer COVID- } 19 \text { en el } \\
\text { peri-operatorio }\end{array}$ & $1(1)$ & $9(6)$ & $30(20)$ & $32(22)$ & $75(51)$ \\
\hline $\begin{array}{l}\text { Temor de los médicos a potenciales demandas de pacientes } \\
\text { infectados por COVID-19 durante el perioperatorio }\end{array}$ & $21(14)$ & $25(17)$ & $41(28)$ & $33(22)$ & $27(19)$ \\
\hline $\begin{array}{l}\text { Dificultad para asegurar de manera sostenida los elementos } \\
\text { de protección personal para el personal en salud }\end{array}$ & $21(14)$ & $23(16)$ & $36(25)$ & $34(23)$ & $33(22)$ \\
\hline $\begin{array}{l}\text { Falta de pruebas diagnósticas para identificar pacientes pro- } \\
\text { gramados a procedimientos electivos que estén infectados por } \\
\text { COVID-19 }\end{array}$ & $15(10)$ & $22(15)$ & $27(18)$ & $30(21)$ & $52(36)$ \\
\hline $\begin{array}{l}\text { Falta de rutas hospitalarias claras para la minimización de } \\
\text { contaminación cruzada entre pacientes COVID-19 y los que no }\end{array}$ & $40(27)$ & $34(23)$ & $24(16)$ & $24(16)$ & $25(17)$ \\
\hline $\begin{array}{l}\text { Normativa gubernamental no clara respecto de las recomen- } \\
\text { daciones para reiniciar procedimientos electivos }\end{array}$ & 19(13) & $25(17)$ & $29(20)$ & $35(24)$ & $39(26)$ \\
\hline $\begin{array}{l}\text { Restricciones vigentes para movilización de pacientes entre } \\
\text { ciudades }\end{array}$ & $18(12)$ & 18(12) & $29(20)$ & $32(22)$ & $50(34)$ \\
\hline $\begin{array}{l}\text { Direccionamiento de los recursos económicos del sistema de } \\
\text { salud para el cuidado COVID-19 con disminución hacia áreas } \\
\text { como electrofisiología }\end{array}$ & $21(14)$ & 19(13) & $34(23)$ & $25(17)$ & $48(33)$ \\
\hline
\end{tabular}

picos máximos de número de casos. Incluso se menciona que parte de la infraestructura dedicada a los SEF se convirtió para atender pacientes COVID-19 ${ }^{(8)}$. El descenso referido por los médicos encuestados no se relacionaba aún con las incidencias más altas que hubo luego en la región. A final de mayo de 2020 la cantidad de casos nuevos diarios por millón de habitantes en Sudamérica era de 85 y en mayo de 2021 ha superado los 300 sin haber descendido nunca de los 100 casos/ diarios. Las realidades particulares de cada país son heterogéneas, pero se acepta que ésta pandemia es un problema de abordaje global $^{(9)}$.

Al momento de la encuesta, el personal médico de los SEF no había sido mayormente afectado por la enfermedad ni contactos. El descenso de la actividad en electrofisiología tampoco se justificaba en una saturación locativa en los centros hospitalarios. El 65\% de los encuestados refirió un grado de ocupación de camas bajo o intermedio. Por otro lado, los médicos mayoritariamente se desplazaban entre varios centros asistenciales y compartían sala de procedimientos con servicios de hemodinamia. Muchos de los encuestados refirieron que aún no se habían tomado varias de las medidas para contener la propagación.

El retorno a la actividad normal es un tema que preocupa hasta hoy. Al momento de la encuesta los electrofisiólogos mayoritariamente (63\%) estaban considerando la reapertura de su servicio, a pesar de que la adherencia a los protocolos de protección no era óptima (67\%) tampoco las pruebas de tamizaje al personal (77\%) y la mitad (53\%) no hacían pruebas de detección previa en los pacientes. Hoy se considera que todos éstos elementos son necesarios para volver a la presencialidad con seguridad ${ }^{(10,11)}$.

La información de esta encuesta permite plantear que, atendiendo a la información que se tenía de Europa y China, los SEF habían restringido mucho su actividad, pero los hospitales no estaban sufriendo aún la carga de enfermedad esperada. Esto se asoció con una visión optimista sobre el futuro cercano, pero la realidad fue que la incidencia de COVID-19 aumentó de forma muy considerable.

Habrá que seguir evaluando lo ocurrido y analizar la situación presente en Latinoamérica, siendo actualmente la región más afectada por la pandemia.

\section{Conclusiones}

En marzo del 2020 hubo una reducción muy im- 
portante de la actividad clínica y de los procedimientos invasivos en electrofisiología. En la mayoría de los SEF no había falta de disponibilidad de camas ni contagios importantes del personal. Muy pocos habían realizado procedimientos a pacientes con COVID-19.

Los electrofisiólogos trabajan mayoritariamente en varios centros y en salas que comparten con servicios de hemodinamia.

En ese momento la tasa de infección era significativamente más baja que la actual y aún no se habían implementado totalmente medidas de prevención, pero existía mayoritariamente la percepción de que en poco tiempo se retomaría la actividad normal.

\section{Limitaciones}

Se analizaron los datos en conjunto. Esto da información del contexto general, pero puede esconder particularidades de algún país y más aún de algunos centros.

No se condicionó las repuestas cuantitativas de los participantes y cada uno pude haberlo hecho de distinta manera y criterio.

Los protocolos de actuación en cada servicio pueden diferir y no fueron evaluados.

La investigación realizada no tomó una muestra representativa de los electrofisiólogos de Latinoamérica. Se superó el $40 \%$ de la población objetivo y hubo participantes de 18 de los 20 países que integran LAHRS. La información obtenida corresponde al momento en que se hizo el relevamiento y no se puede inferir de aquí el comportamiento posterior.

\section{Agradecimientos}

Al Sr. Alejandro Juan por su apoyo en el área de informática.

Al Lic. Soc. Martín García por su apoyo en la interpretación de los resultados.

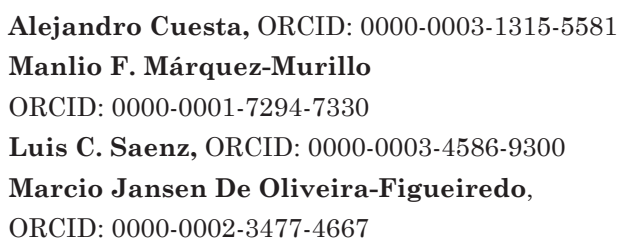

Este artículo fue aceptado para su publicación por el Editor asociado, Dr. Ignacio Batista

\section{Bibliografía}

1. Shereen MA, Khan S, Kazmi A, Bashir N, Siddique R. COVID-19 infection: Origin, transmission, and characteristics of human coronaviruses. J Adv Res 2020;24:91-98. doi: 10.1016/j.jare.2020.03.005.

2. Yang J, Zheng Y, Gou X, Pu K, Chen Z, Guo $\mathbf{Q}$, et al. Prevalence of comorbidities and its effects in patients infected with SARS-CoV-2: a systematic review and meta-analysis. Int J Infect Dis 2020;94:91-95. doi: 10.1016/j.ijid.2020.03.017.

3. Clerkin KJ, Fried JA, Raikhelkar J, Sayer G, Griffin JM, Masoumi A et al. COVID-19 and Cardiovascular Disease. Circulation. 2020;141(20):1648-1655. doi: 10.1161/CIRCULATIONAHA.120.046941. Epub 2020 Mar 21. PMID: 32200663.

4. Lakkireddy DR, Chung MK, Gopinathannair R, Patton KK, Gluckman TJ, Turagam M, et al. Guidance for cardiac electrophysiology during the COVID-19 pandemic from the Heart Rhythm Society COVID-19 Task Force; Electrophysiology Section of the American College of Cardiology; and the Electrocardiography and Arrhythmias Committee of the Council on Clinical Cardiology, American Heart Association. Circulation. 2020;141(21):e823-e831. doi: 10.1161/CIRCULATIONAHA.120.047063.

5. Saenz LC, Miranda A, Speranza R, Texeira RA, Rojel U, Enriquez A et al. Recommendations for the organization of electrophysiology and cardiac pacing services during the COVID-19 pandemic : Latin American Heart Rhythm Society (LAHRS) in collaboration with: Colombian College Of Electrophysiology, Argentinian Society of Cardiac Electrophysiology (SADEC), Brazilian Society Of Cardiac Arrhythmias (SOBRAC), Mexican Society Of Cardiac Electrophysiology (SOMEEC). J Interv Card Electrophysiol. 2020;59(2):307-13. doi: 10.1007/s10840-020-00747-5.

6. Varma N, Marrouche NF, Aguinaga L, Albert CM, Arbelo E, Choi JI et al. HRS/EHRA/ APHRS/LAHRS/ACC/AHA Worldwide Practice Update for Telehealth and Arrhythmia Monitoring During and After a Pandemic. J Am Coll Cardiol. 2020;76(11):1363-74. doi: 10.1016/j. jacc.2020.06.019.

7. Li J, Mazzone P, Leung LWM, Lin W, D'Angelo G, Ma J et al. Electrophysiology in the time of coronavirus: coping with the great wave. Europace. 2020;22(12):1841-7. doi: 10.1093/europace/euaa185. PMID: 32995866; PMCID: PMC7543596.

8. Mazzone P, Peretto G, Radinovic A, Limite LR, Marzi A, Sala S et al. The COVID-19 challenge to cardiac electrophysiologists: optimizing resources at a referral center. J Interv Card Electrophysiol. 2020:1-7. doi: 10.1007/s10840-020-00761-7.

9. Ritchie H, Mathieu E, Rodés-Guirao L, Appel C, Gattino Ch, Ortiz-Ospina E, et al. Coronavirus (COVID19) cases [Internet]. Oxford: Our World in Data;2020 [consulta: 21 May 2021]. Disponible 
en: https://ourworldindata.org/covid-cases

10. Barnes M, Sax PE. Challenges of "Return to Work" in an Ongoing Pandemic. N Engl J Med. 2020;383(8):779-786. doi: 10.1056/NEJMsr2019953.

11. Wood DA, Mahmud E, Thourani VH, Sathananthan J, Virani A, Poppas A et al. Safe
Reintroduction of Cardiovascular Services During the COVID-19 Pandemic: From the North American Society Leadership. J Am Coll Cardiol. 2020;75(25):3177-83. doi: 10.1016/j. jacc.2020.04.063.

Contribución de autores

Alejandro Cuesta. Ejecución de la encuesta, análisis y redacción del manuscrito.

Manlio F. Márquez-Murillo. Difusión, redacción del manuscrito y corrección.

Luis C. Saenz. Confección de la encuesta, difusión y corrección del manuscrito.

Marcio Jansen De Oliveira-Figueiredo. Difusión, corrección del manuscrito y redacción del resumen en portugués. 